\title{
Development of Computational Technique for Labeling Magnetic Flux-Surfaces
}

\author{
Masanori NUNAMI ${ }^{1)}$, Ryutaro KANNO ${ }^{1)}$, Shinsuke SATAKE ${ }^{1)}$, \\ Hisanori TAKAMARU ${ }^{2)}$ and Takaya HAYASHI ${ }^{1), 3) *}$ \\ ${ }^{1)}$ National Institute for Fusion Science, Toki 509-5292, Japan \\ ${ }^{2)}$ Department of Computer Science, Chubu University, Kasugai 487-8501, Japan \\ ${ }^{3)}$ Department of Fusion Science, Graduate University for Advanced Studies, Toki 509-5292, Japan
}

(Received 3 March 2006 / Accepted 14 June 2006)

\begin{abstract}
In recent Large Helical Device (LHD) experiments, radial profiles of ion temperature, electric field, etc. have been measured in the $m / n=1 / 1$ magnetic island produced by island control coils, where $m$ is the poloidal mode number and $n$ the toroidal mode number. When the plasma transport in radial profiles is numerically analyzed, an average over a magnetic flux-surface in the island is a very useful concept to understand the transport. When averaging, a proper labeling of the flux-surfaces is necessary. In general, it is not easy to label the flux-surfaces in a magnetic field containing the island, compared with the case of a magnetic field configuration having nested flux-surfaces. In the present paper, we have developed a new computational technique to label the magnetic flux-surfaces. This technique uses an optimization algorithm called the simulated annealing method. The fluxsurfaces are discerned by using two labels: one is classification of the magnetic field structure, i.e., core, island, ergodic, and outside regions, and the other depends on the value of the toroidal magnetic flux. We have applied this technique to an LHD configuration with the $m / n=1 / 1$ island, and successfully discriminated of the magnetic field structure.
\end{abstract}

(c) 2006 The Japan Society of Plasma Science and Nuclear Fusion Research

Keywords: Large Helical Device, magnetic island, flux-surface, Poincaré plots, annealing method

DOI: $10.1585 /$ pfr. 1.038

\section{Introduction}

A flux-surface average of some quantity, e.g., particle flux, heat flux, etc., is a very useful concept in the transport analysis of a toroidal plasma. The flux-surface average of a function $\Phi(\boldsymbol{x})$ is defined by the volume average over an infinitesimally small shell with volume $\Delta V$, where $\Delta V$ lies between two neighboring flux-surfaces with volumes $V$ and $V+\Delta V$, and is denoted as

$$
\langle\Phi(\boldsymbol{x})\rangle=\lim _{\Delta V \rightarrow 0} \frac{1}{\Delta V} \iiint_{\Delta V} \Phi(\boldsymbol{x}) \mathrm{d}^{3} x,
$$

if there exist closed flux-surfaces. When averaging, we have to label the magnetic flux-surfaces; e.g., if nested flux-surfaces exist, the surface is labeled by the volume enclosed by the surface in the Hamada coordinates [1], and by the toroidal magnetic flux in the Boozer coordinates $[2,3]$. In a non-axisymmetric configuration, the existence of nested flux-surfaces is not guaranteed [4]. However, it is possible to numerically obtain an MHD equilibrium having closed magnetic surfaces by using a suitable three-dimensional equilibrium code which does not assume the existence of nested flux-surfaces, e.g., the HINT code [5-7]. In general, such an equilibrium includes magnetic islands and ergodic regions. Thus, magnetic flux-

author's e-mail: nunami.masanori@nifs.ac.jp

* Deceased. surfaces are not easily labeled. Without any approximations, we cannot evaluate flux-surface averages in terms of magnetic coordinates in a non-axisymmetric configuration. Although quasi magnetic-coordinates can be constructed on the equilibrium with the islands as shown in Refs. [8-12], such a coordinate system does not correspond to a magnetic coordinate system along the fluxsurfaces of the islands.

In recent Large Helical Device (LHD) experiments, radial profiles of ion temperature, electric field, etc. have been measured in the $m / n=1 / 1$ magnetic island produced by island control coils $[13,14]$. Numerical transport analysis is required for understanding the experimental results; thus, the average over the island's flux-surface is necessary.

In the present paper, we develop a new computational technique for labeling magnetic surfaces in the LHD configuration. The flux-surfaces can be labeled by tracing field lines, as shown in Refs. [15-18]. The main task of the technique is to identify a flux-surface from the Poincaré plots of a field line on a poloidal cross section. For the identification, the points of the Poincaré plots, i.e., the Poincaré points, should be numbered along a closed curve given by the poloidal cross section of a flux-surface; in the present paper this procedure is called the ordering of the Poincaré points. One of the simplest methods for ordering the Poincaré points is to search for each point's nearest 
neighboring point. However, this method often fails at, for example, a closed magnetic surface on the island. In order to improve the ordering procedure, we employ an algorithm called the simulated annealing method [19,20]. This method is familiar as a successful algorithm to solve the traveling salesman problem [21], which is a problem involving a traveling salesman who has to visit a number of cities, and how to plan the trip so that every city is visited once and just once while making the whole trip as short as possible. This method is useful for solving our problem, i.e., how to connect each Poincaré point of a field line. In the technique developed here, the magnetic flux-surfaces are identified by two labels, IREGION and TFLUX, which describe the classification of a magnetic field structure and a toroidal magnetic flux, respectively.

When the number of sampling flux-surfaces is quite large, a calculation code based on the developed technique should be parallelized. The code has been programed us- ing High Performance FORTRAN [22] on a vector parallel supercomputer.

This paper is organized as follows. In Sec. 2, we show the outline of the developed technique which is composed of three parts shown in Secs. 2.1-2.3; the numerical results are shown in Sec. 2.4. Finally, a summary is given in Sec. 3.

\section{Computational Technique}

We explain the outline of the technique as follows. Figure 1 shows a flowchart of the developed technique. As seen in the flowchart, the technique is constructed of three parts: I) Classification of regions, II) Ordering of Poincaré points, and III) Calculation of toroidal magnetic flux. In part I, for a given magnetic field configuration, we classify regions of the magnetic field structure, i.e., core, island, ergodic, and outside regions. In part II, we trace a field line

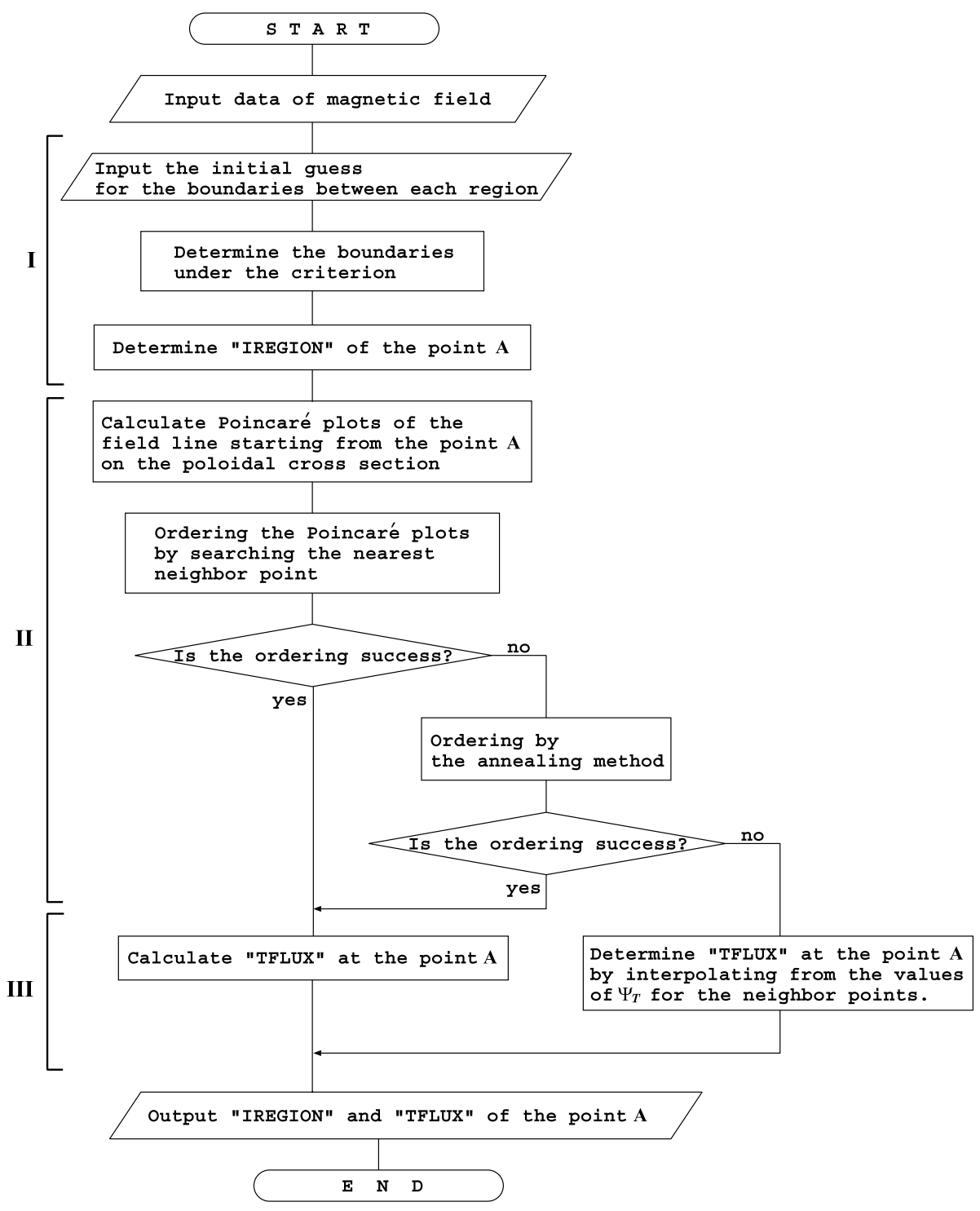

Fig. 1 Flowchart of the developed technique. The chart describes the procedure for labeling a flux-surface which includes an evaluation point $A$. This technique is grouped into three parts: I) classification of regions, II) ordering Poincaré points, and III) calculation of toroidal magnetic flux, where IREGION is the label of the regions and TFLUX is the value of the toroidal flux. 
to obtain the Poincaré points on a poloidal cross section, and order these points by using two methods explained in Sec. 2.2. In part III, we calculate a value of the toroidal magnetic flux for the closed magnetic flux-surface represented by the Poincaré points, and label it by the value.

\subsection{Classification of regions}

In this section, we consider the classification of regions of the magnetic field structure. Because there does not exist a magnetic coordinate system along the closed magnetic surfaces in both core and island regions, we have to classify the regions, as shown in Fig. 2. We can discern four parts of the structure, i.e., outside, ergodic, island, and core regions. Here, we consider that the $m / n=1 / 1$ island is visible under certain numerical accuracy, while the other islands are not. Similarly, the ergodic region is considered to be visible but narrow.

In general, it is not easy to numerically recognize the topology of a magnetic field structure, which is characterized as the four regions mentioned above, without preliminary knowledge of the structure. Therefore, first of all, in order to guess the topology, we calculate the Poincaré plots of field lines on a poloidal cross section; for example, we trace the field lines having their starting points at certain regular intervals on $Z=0$ axis. We carefully pick three flux-surfaces from the Poincaré points in order to obtain an initial guess of three boundaries between the outside and ergodic regions, island and ergodic regions, and inside and ergodic regions. Thus, the topology of the structure is defined in the above way under the accuracy of the intervals between magnetic flux-surfaces given by the Poincaré plots, and more precise topology of the structure is neglected. Note that the above procedure for the topol-

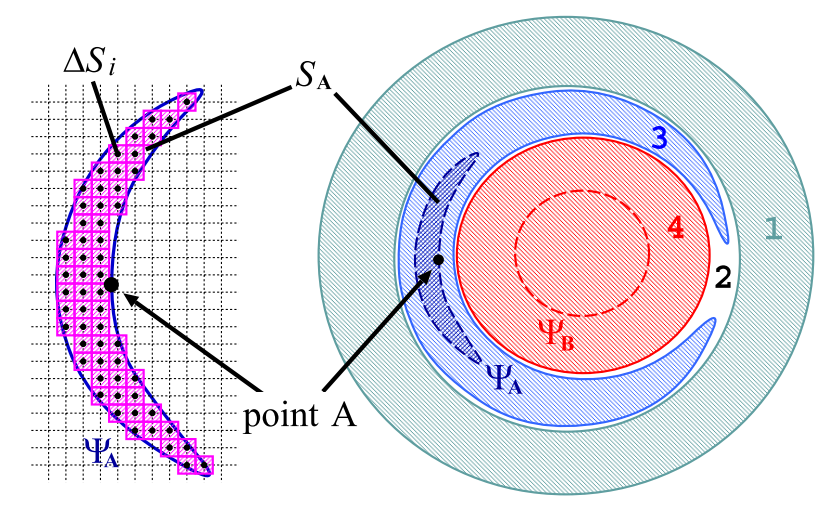

Fig. 2 Classification of the regions (right-hand) and illustration of the calculation of the toroidal magnetic flux (lefthand). We classify the regions into four parts: outside, ergodic, island, and core regions by the label IREGION $=1,2,3$, and 4 , respectively. A magnetic coordinate system does not exist along both the closed magnetic flux-surfaces, $\Psi_{\mathrm{A}}$ and $\Psi_{\mathrm{B}}$. For calculating the toroidal flux TFLUX at the evaluation point $A$, we sum the toroidal fluxes $B_{\varphi}^{(i)} \Delta S_{i}$ over the interior region $S_{\mathrm{A}}$ of $\Psi_{\mathrm{A}}$. ogy is a premise for a user of the developed calculation code, i.e., the user already knows the topology but is not required to have precise information regarding the boundaries between each region. Next, in order to determine the boundaries, we input the initial guess of the boundaries obtained above into the calculation code, and the code starts searching the boundaries from the initial guess. The code numerically determines the boundaries by finding a fluxsurface which encloses a maximum area of each region. This procedure is performed as follows. In order to judge whether or not a flux-surface can be constructed, the code orders the Poincaré points of the flux-surface and checks for the nonexistence of a crossing path between any points. The details of ordering Poincaré points and treatment of crossing paths will be shown in the following sections. The code then checks a neighboring flux-surface by shifting the starting point of Poincaré points calculation toward the direction in which the area of each region becomes larger. Note that when a flux-surface is a rational surface in each region, there may exist crossing paths even if we use the ordering procedure. In such a case, the code searches for other points which can increase the region's area. Thus, by representing the operation, the code determines the boundaries enclosing the maximum area of each region. We presuppose that each region, i.e., inside, outside, and island regions, consists of nested flux-surfaces under the accuracy considered here.

By using the determined boundaries, we classify the regions into four parts; IREGION = 1 (outside region), 2 (ergodic region), 3 (island region), and 4 (core region), as shown in Fig. 2.

\subsection{Ordering Poincaré points with annealing method}

Next, the Poincaré points of a field line must be ordered on a poloidal cross section in order to identify the closed magnetic flux-surface. Note that in an ergodic region, a visible flux-surface does not exist, thus field line tracing is not performed in such a region. For ordering, we use two methods. One is a simple method that orders the points by searching for each point's nearest neighboring point, where the ordering begins from a given starting point of the Poincaré points. This is a simple ways of ordering the points. But we frequently encounter a case in which this simple method does not work well, as will see later. The other method is based on the simulated annealing method $[19,20]$. This method is a well-known optimization algorithm employed in the traveling salesman problem [21] (the problem of finding the shortest cyclical itinerary for a traveling salesman who must visit each of $N$ cities in turn). We use it to order the Poincaré points, when the simple method does not perform effectively (see part II of Fig. 1). In the following, we provide a brief description of the simulated annealing method used for solving our problem. 


\subsubsection{Simulated annealing method}

The simulated annealing method is a probabilistic algorithm for combinatorial optimization problems [23]. In particular, when a given function has many local extrema, the method is powerful for searching the global extremum. Of course, the most sure method for searching the global extremum is to search for all possibilities; e.g., in the traveling salesman problem, the most sure method searches the true route with the minimum length from all possible routes. In such a method, we can always find the true extremum. But this method is extremely time-consuming; for example, in the traveling salesman problem involving $N$ cities, the calculation time increases as $\sim \exp (N)$ [23]. Note that the most sure method is completely different from the simple method explained above, because the starting point for searching the true route in the simple method is fixed. On the other hand, the simulated annealing method can give us a better solution under realistic calculation costs. Our main aim in this section is to identify a poloidal cross section of a flux-surface by using the Poincare points of the field line. We assume that the cross section of the flux-surface is obtained by connecting the points via the shortest route. This assumption is not always guaranteed to identify the flux-surface, but it is almost valid for practical cases, as shown in Sec. 2.2.3.

The simulated annealing method is formulated by analogy with the annealing of a heated metal with lattice defects, under slow cooling [19]. As the temperature decreases slowly, the heated metal forms a pure crystal which is the material's lowest energy state. On the other hand, if the temperature decreases rapidly, it forms a non-crystal which is not the lowest energy state. This analogy suggests us that the lowest energy state, i.e., the global minimum of its energy function, is obtained by slow cooling. An energy equilibrium with a temperature $T$ is distributed with a Boltzmann probability distribution,

$$
P(E) \sim \exp \left(-\frac{E}{k T}\right),
$$

where $k$ means the Boltzmann constant. According to the probability $P(E)$, a lower energy state can climb up to a higher energy state, i.e., there is a possibility that the system can escape from a local minimum of the energy to a better state. As shown in Fig. 3, when the system is trapped in a local minimum of the function, it can escape with the probability $P \sim \exp \left[-\left(E_{2}-E_{1}\right) / T\right]$, where the energy of the system is changed from $E_{1}$ to $E_{2}\left(>E_{1}\right)$. As the temperature parameter $T$ decreases slowly, the probability $P$ becomes small. We may then reach the function's global minimum. In the following, we show how the method works, with a simple example.

\subsubsection{Illustration of annealing method}

Here we illustrate the annealing method applied to the traveling salesman problem. The $N$ points are provided as shown in Fig. 4, where the number of each point describes

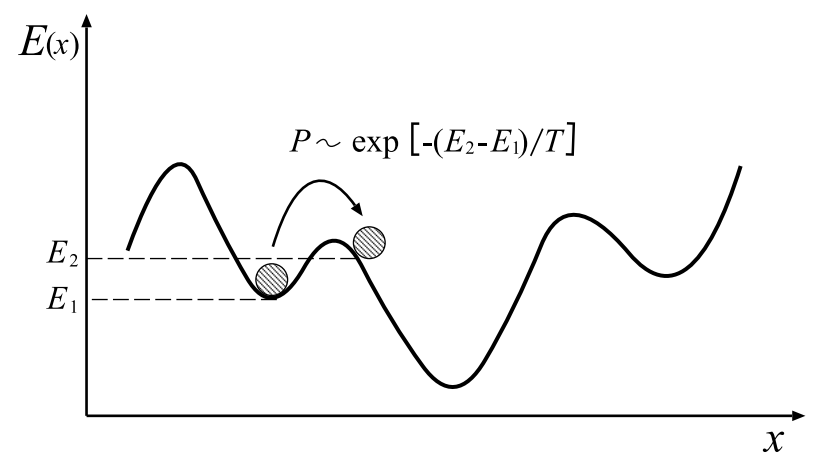

Fig. 3 Escape from a local minimum. When the system is trapped in a local minimum $E_{1}$, it can escape from there, according to the probability $P \sim \exp \left[-\left(E_{2}-E_{1}\right) / T\right]$.

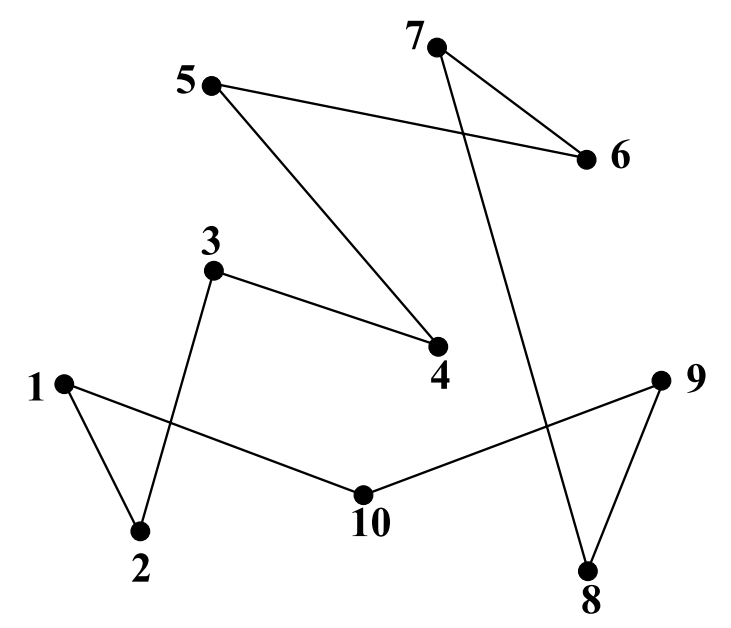

Fig. 4 The initial route of the traveling salesman problem. The numbers describe the initial order.

the initial order $i=1, \ldots, N, N=10$ as seen in Fig. 4 . Each point is located at $\left(x_{i}, y_{i}\right)$ and the solid line in the figure represents the initial route of the salesman. An arrangement is defined as a permutation of the number $1, \ldots, N$, interpreted as the ordering in which the points are visited.

The initial order is rearranged as follows. We randomly choose a segment from the initial route for the rearrangement. For example, in Fig. 5, a segment of the route is chosen as the red line, where $n_{1}$ is the beginning of the segment and $n_{2}$ the end of the segment. Here, this segment is named $n_{1}-n_{2}$. We introduce an objective function $E$ to estimate a degree of the optimization. This function can be defined in various forms according to the considered cases. In our problem, the objective function is given as the total length of the route,

$$
E=\sum_{i=1}^{N} L(i, i+1),
$$

where the point $i=N+1$ is identified with the point $i=1$, and $L(j, k)$ is defined to represent a path-length between a 


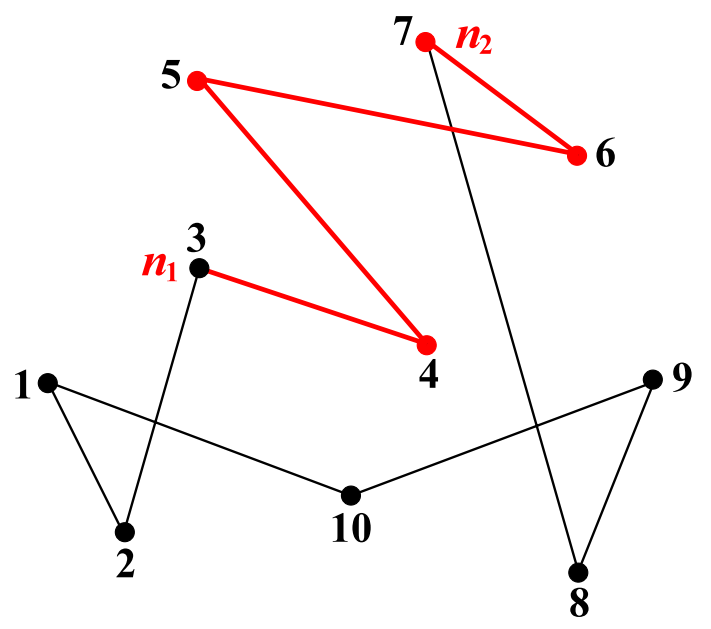

Fig. 5 The choice of the segment for the rearrangement. In this case, the segment $n_{1}-n_{2}$ indicated by the red line is the target for the rearrangement operations, i.e., reversal and transplant.

point $j$ and a point $k$; i.e.

$$
L(j, k):=\sqrt{\left(x_{j}-x_{k}\right)^{2}+\left(y_{j}-y_{k}\right)^{2}} .
$$

For finding the solution of the problem, the function $E$ should be minimized. In the method, we rearrange the order of the points in either of the following two ways, reversal or transplant, which have the same probability.

The reversal operation involves the removal of the segment and its replacement with the same points in the opposite order. As shown in Fig. 6, we reverse the segment $n_{1}-n_{2}$; i.e., we connect $n_{1}$ to $n_{4}$, and $n_{2}$ to $n_{3}$. In the figure, the blue lines represent new paths after reversing the segment. We then introduce the cost of the reversal operation, $C_{\text {rev }}$, defined by the difference between the total lengths of the route before and after the reversal operation; i.e., $C_{\text {rev }}=E_{\text {after }}-E_{\text {before }}$. In the case of Fig. 6, considering only changing paths, the cost of the reversal is

$$
\begin{aligned}
C_{\text {rev }} & =[L(2,7)+L(3,8)] \\
& -[L(2,3)+L(7,8)] .
\end{aligned}
$$

The other method of rearranging the order of the points is the transplant operation in which the segment is removed and transplanted between two neighboring points which are randomly chosen from the points not on the segment. In Fig. 7, the destination path of the transplant, $n_{3}$ $n_{4}$, is chosen. We transplant the segment $n_{1}-n_{2}$ into the destination path $n_{3}-n_{4}$, and then close the route. In the figure, the dashed-lines represent the old paths which are replaced by the blue paths after transplanting the segment $n_{1}-n_{2}$. The cost of the transplant operation, $C_{\mathrm{tr}}$, is given as

$$
\begin{aligned}
C_{\mathrm{tr}} & =[L(2,8)+L(9,7)+L(3,10)] \\
& -[L(2,3)+L(7,8)+L(9,10)] .
\end{aligned}
$$

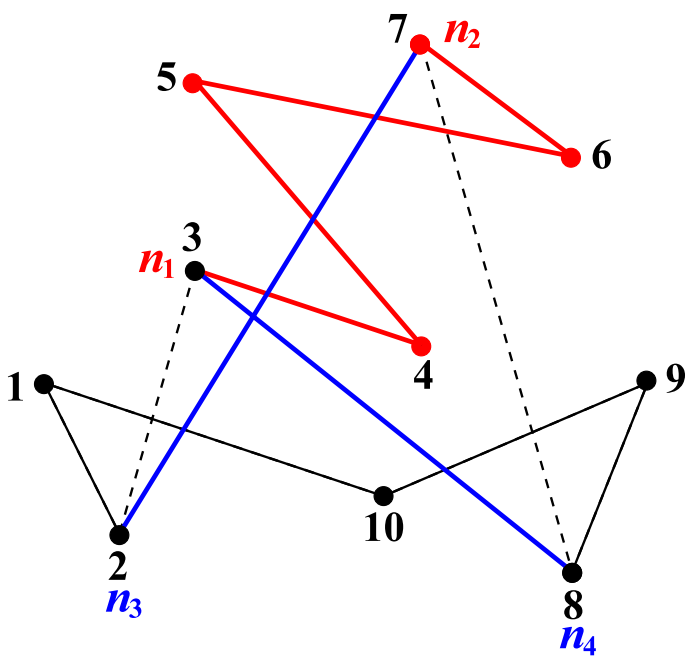

Fig. 6 Reversal of the segment $n_{1}-n_{2}$. In the reversal operation, the segment $n_{1}-n_{2}$ is reversed; i.e., the beginning point $n_{1}$ of the segment is connected to the point $n_{4}$, and the end point $n_{2}$ to the point $n_{3}$. Dashed lines represent the old paths before the reversal operation.

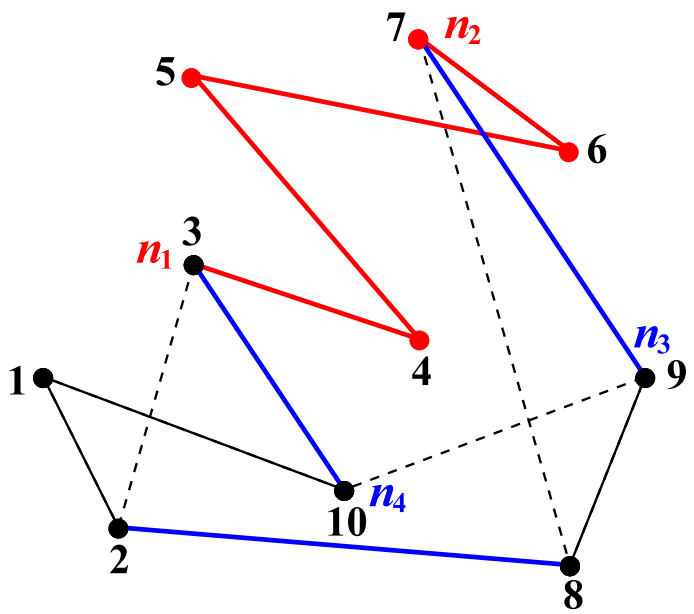

Fig. 7 Transplant of the segment $n_{1}-n_{2}$. In the transplant operation, the segment $n_{1}-n_{2}$ is transplanted into $n_{3}-n_{4}$ which is randomly chosen; i.e., the beginning point $n_{1}$ is connected to $n_{4}$, and the end point $n_{2}$ to $n_{3}$. Point 2 has to be connected to point 8 in order to close the route. Blue lines represent new paths in the route, and dashed lines represent the old paths before the transplant operation. By repeating the transplant operation (this figure) and the reversal operation (Fig. 6), the re-ordering of the points is executed.

After estimating the cost of the reversal or transplant operation, we adopt the rearrangement according to the probability $P_{\mathrm{A}}$ based on the Metropolis algorithm [24], where $P_{\mathrm{A}}$ is defined by the cost $C$ and the temperature $T$,

$$
P_{\mathrm{A}}:=\left\{\begin{array}{ll}
1 & \text { for } C \leq 0 \\
\exp (-C / T) & \text { for } C>0
\end{array} .\right.
$$

By the above operations, the annealing, i.e., escape with 
the Boltzmann probability distribution $P(E)$ in Eq. (2), is executed.

We proceed through the above steps, i.e., the repetition of the rearrangements and the annealing operations, according to an annealing schedule which controls the procedure for reducing the temperature parameter $T$ [23]. When efforts to reduce the total path-length $E$ become sufficiently discouraging, the all calculation steps are stopped.

\subsubsection{Demonstration and application of annealing method}

We demonstrate the annealing method using an example of the traveling salesman problem. As shown in Fig. 8, an initial set of 30 random points is given, where the number means the initial order in which the points are visited, and the solid lines represent the initial route of the salesman. The total length of the initial route is calculated as $E_{\text {initial }}=18.16521$. The results of the simple method for searching the nearest neighboring point and the simulated annealing method are shown in Figs. 9 and 10 , respectively. Each route's total length is calculated as $E_{\text {nearest }}=4.994745$ and $E_{\text {anneal }}=4.546217$. Using the simulated annealing method, we obtain better solution for optimization of the cyclical itinerary. This means that when the simple method is used, the system is trapped in a local minimum of the total length $E$, as shown in Fig. 3, because of the wrong choice of initial starting point. Therefore, if the simple method does not function effectively as seen in such a situation, we have an alternative way, i.e., the simulated annealing method, to obtain the solution of the traveling salesman problem.

We apply the annealing method to order the Poincaré points of a field line. Specifically, we consider that the route connecting the points, which is obtained by using the

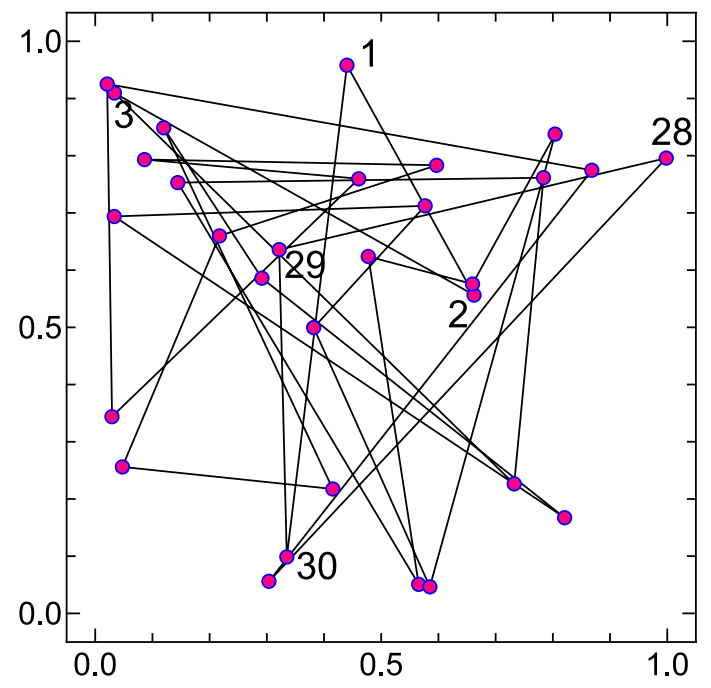

Fig. 8 The initial set of 30 points. The numbers describe the initial order and the solid line represents the initial route. The total length of the initial route is $E_{\text {initial }}=18.16521$. annealing method, is the closed curve of a poloidal cross section of a magnetic flux-surface. Note that the minimum of $E$ is not always guaranteed to identify a flux-surface; in general, when the total number of points is small, many possible routes can be considered for lack of sufficient information regarding the surface, and the shortest route does not always express the flux surface's true curve. If the number of points is sufficiently large, then the flux-surface is usually identified by the minimum of $E$.

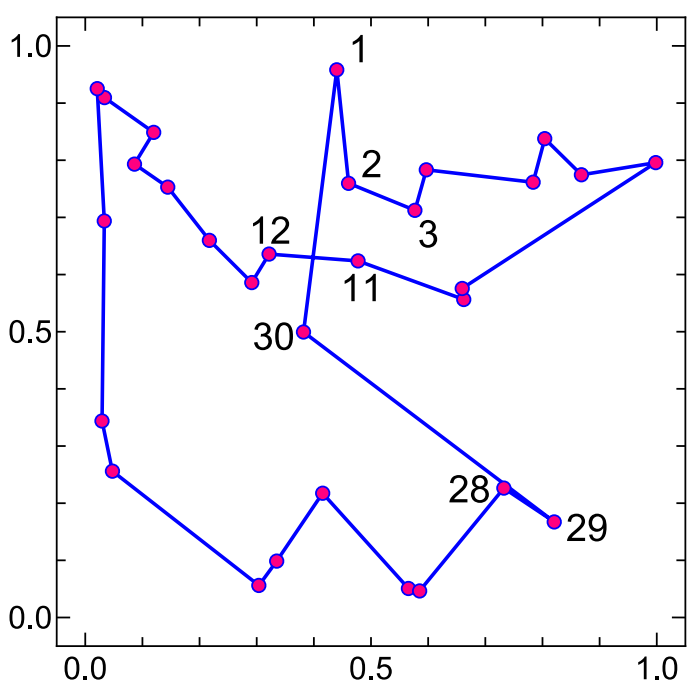

Fig. 9 Result given by the simple method searching the nearest neighboring point. The ordering fails, because the path from point 30 to point 1 crosses the path from 11 to 12 . The total length of the route is $E_{\text {nearest }}=4.994745$.

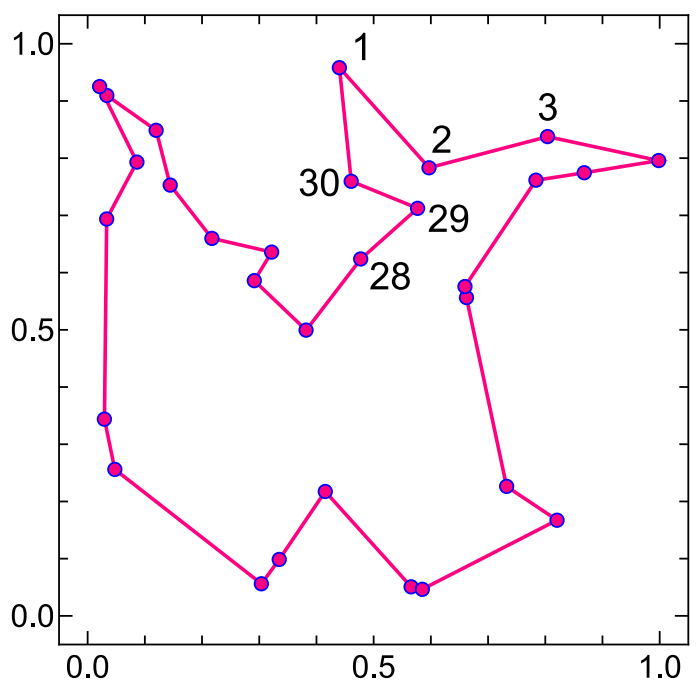

Fig. 10 The result given by the simulated annealing method. The ordering succeeds, because all paths from point $i$ to point $i+1$ do not cross each other, where $i=1,2, \ldots, 30$, and point 31 is identified as point 1 . The solid line may be a better minimum solution of the total length of the route. In fact, the total length is $E_{\text {anneal }}=4.546217$, which is less than the result of the simple method shown in Fig. 9. 

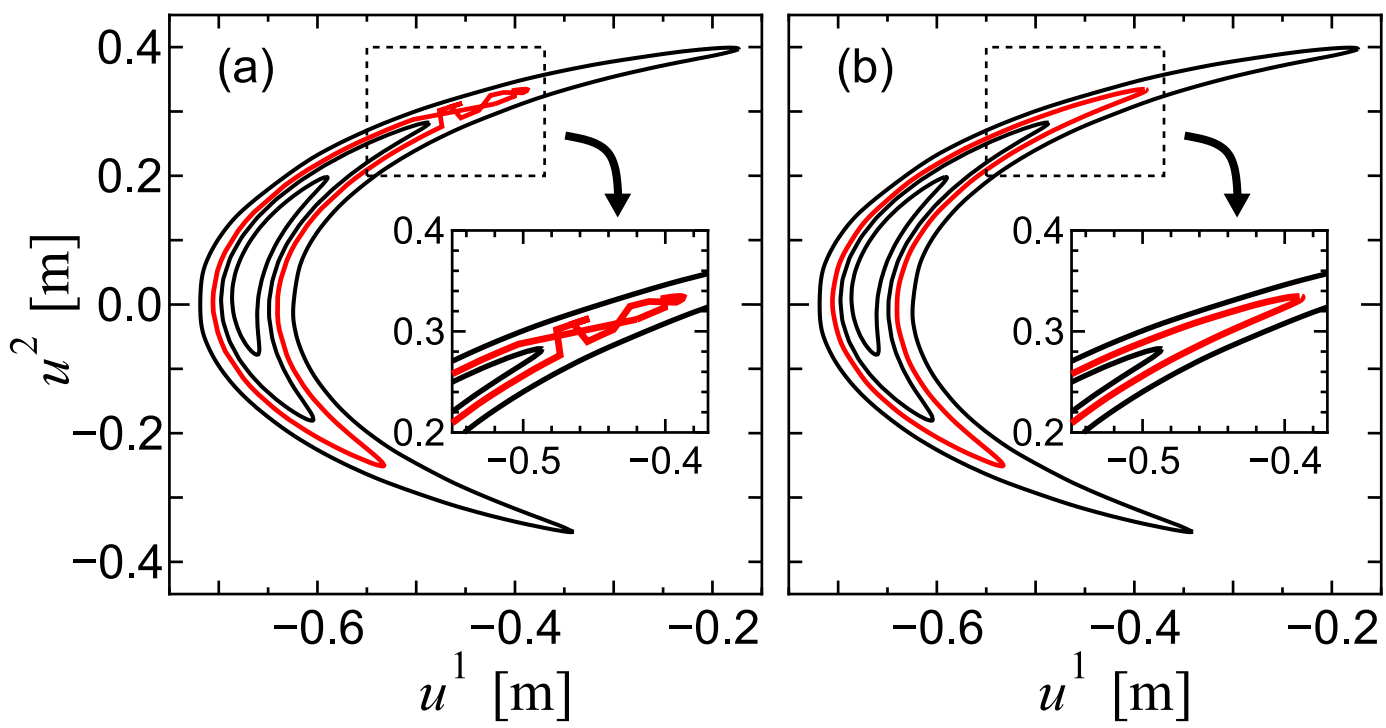

Fig. 11 Comparison between the results of the two ordering methods, i.e., the simple method and the simulated annealing method, in an LHD configuration with an $m / n=1 / 1$ island. The ordering by the simple method is unsuccessful; see the red line in figure (a). On the other hand, the simulated annealing method succeeds; see figure (b).

As shown in Fig. 11 (a), the simple method of searching the nearest neighbors often fails the ordering. Of course, if the number of the points becomes extremely large, then the simple method may succeed to obtain the flux-surface, but it is very time consuming. As shown in Fig. 11 (b), the annealing method can find the flux-surface using an amount of computing time comparable to the case of Fig. 11 (a). Therefore, the annealing method enables us to obtain a flux-surface within a realistic calculation time. Note that it is difficult to identify a rational surface by using the methods introduced here, because in this case the number of their Poincare points is not sufficient to cover the surface, and crossing paths may be caused by insufficient information regarding the surface guessed from the points. In such a case, we define the rational surface by interpolating from neighbor irrational surfaces (see part III of Fig. 1).

\subsection{Calculation of toroidal fluxes}

In the last part of the developed technique, we calculate a value of toroidal magnetic flux $\Psi_{\mathrm{T}}$ and assign the label TFLUX to the flux-surface. In the following, we consider the case of $\Psi_{\mathrm{A}}$ in Fig. 2. As shown in the figure, we assign square areas $\Delta S_{i}$ to each grid-point $\left(x_{i}, y_{i}\right)$ which is included in the interior region $S_{\mathrm{A}}$ on a poloidal cross section. Note that the accuracy of the calculation depends on the number of grids. The toroidal flux for the point $A$ in Fig. 2, for example, is written as

$$
\Psi_{\mathrm{T}}=\int_{S_{\mathrm{A}}} B_{\varphi} \mathrm{d} S,
$$

where $B_{\varphi}$ is the toroidal component of the magnetic field $\boldsymbol{B}$. We substitute the value of the magnetic field at the gridpoint $\left(x_{i}, y_{i}\right), \boldsymbol{B}^{(i)}$, for the value in a corresponding area
$\Delta S_{i}$. The toroidal component of $\boldsymbol{B}^{(i)}$ is denoted by $B_{\varphi}^{(i)}$. Thus we numerically proceed to calculate toroidal flux by the sum of $B_{\varphi}^{(i)} \Delta S_{i}$,

$$
\frac{\Psi_{\mathrm{T}}}{\Psi_{\mathrm{N}}} \simeq \frac{\sum_{\left(x_{i}, y_{i}\right) \in S_{\mathrm{A}}} B_{\varphi}^{(i)} \Delta S_{i}}{\Psi_{\mathrm{N}}} \equiv \mathrm{TFLUX},
$$

where $\Psi_{\mathrm{N}}$ is the normalization and TFLUX is the label denoting the value of the toroidal flux. Of course, we can improve the integration scheme more precisely, although we use the simple scheme described above.

If an evaluation point is located on a rational surface or an invisibly thin island, we calculate the toroidal fluxes at the neighboring points of the original point, and interpolate these calculation into the original point, as mentioned in part III of Fig. 1.

\subsection{Numerical results}

In order to check the convergence of the toroidal flux calculation, our result is compared with analytic value of $\Psi_{\mathrm{T}}$ in the magnetic field of a simple tokamak configuration given as

$$
\begin{aligned}
& B_{R}=-\frac{B_{0} R_{0}}{q} \frac{Z}{R^{2}}, \\
& B_{\varphi}=-B_{0} R_{0} \frac{1}{R} \\
& B_{Z}=\frac{B_{0} R_{0}}{q} \frac{\left(R-R_{0}\right)}{R^{2}},
\end{aligned}
$$

in terms of the cylindrical coordinate system, $(R, \varphi, Z)$, where $B_{0}$ is the strength of the magnetic field at the magnetic axis, $R_{0}$ the major radius of the axis, and $q$ a safety factor. It is confirmed that the relative error of our result with the analytic value becomes sufficiently small $\left(\lessgtr 10^{-4}\right)$ 
as the number of the grids increases. The relative error does not depend significantly on the choice of a fluxsurface except a rational surface.

We show the results for labeling the magnetic fluxsurfaces in certain magnetic field configurations. In Fig. 12, we label the flux-surfaces on a poloidal section of a magnetic configuration given by adding an $m / n=$ $1 / 1$ magnetic island to the simple tokamak configuration, which is referred to in this paper as the test configuration. Here, we have used Eulerian coordinates, i.e., the helical coordinate system [6]. The relation between the coordinates $\left(u^{1}, u^{2}, u^{3}\right)$ and the Cartesian coordinates $(X, Y, Z)$ is given as,

$$
\begin{aligned}
& X=\left(R_{0}+u_{*}^{1} \cos h u^{3}+u^{2} \sin h u^{3}\right) \cos u^{3}, \\
& Y=-\left(R_{0}+u_{*}^{1} \cos h u^{3}+u^{2} \sin h u^{3}\right) \sin u^{3}, \\
& Z=-u_{*}^{1} \sin h u^{3}+u^{2} \cos h u^{3},
\end{aligned}
$$

with $u_{*}^{1}=u^{1}+\delta$, where $R=\sqrt{X^{2}+Y^{2}}$ and $\varphi=-u^{3}$. Here, $h$ is the rotation number along the $u^{3}$ direction and $\delta$ the deviation of the geometrical axis of the helical coordinates. In this paper, we set the values as $h=0$ and $\delta=0$. The label of the regions of the magnetic field structure IREGION and the label of toroidal fluxes TFLUX are indicated by colors and their hues, respectively. Here, we consider the success rate of ordering the Poincaré points through the use of the simple method. The rate is proportional to the number of flux-surfaces successfully identified; i.e., there are no crossing paths in the ordering and the points are not located on a rational surface or on an in-

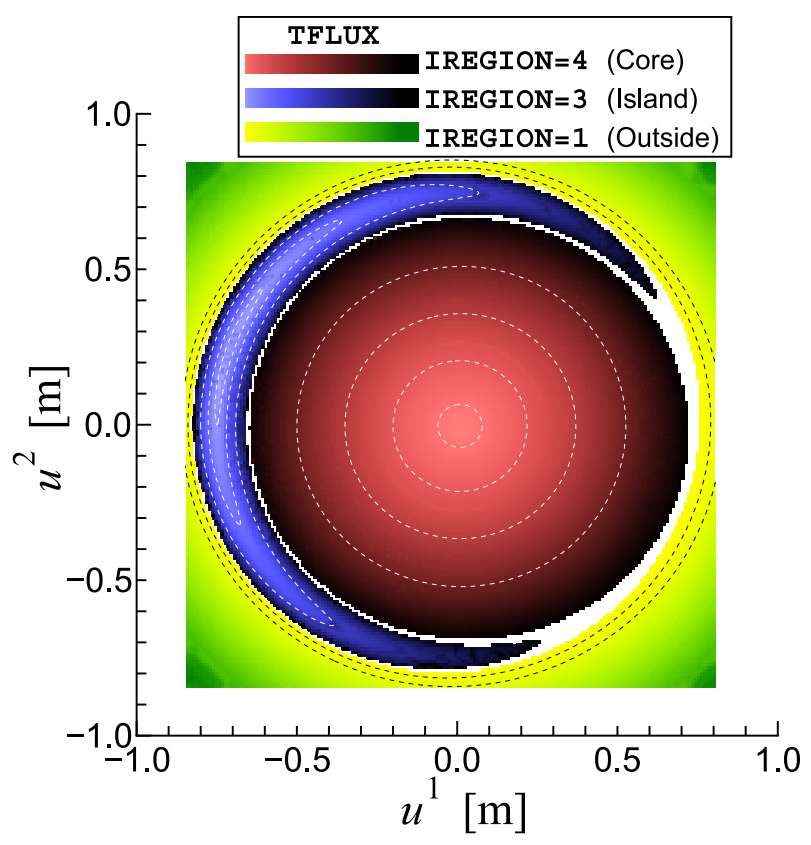

Fig. 12 Label of the magnetic flux-surfaces in the test configuration on a poloidal section $\left(u^{3}=0\right)$, where IREGION and TFLUX are indicated by colors and their hues, respectively. visibly thin island. Table 1 shows the success rates of the simple method in the $m / n=1 / 1$ magnetic island of the test configuration. From the table, we see that as the number of points increases, the rate for the simple method approaches $100 \%$, while the rate for the developed method is always $100 \%$ for all cases shown in the table. As seen in Fig. 13, when the number of the points is small, the simple method often fails, because there is a case in which the path-length in the true curve $L(i, j)$ is larger than the path-length in the less efficient curve $L(i, k)$, i.e., $L(i, j)>L(i, k)$. Thus, the simple method selects a wrong point, as shown in Fig. 13. We should note that there exist some rational surfaces and invisibly thin islands in this configuration. When we encounter points on a rational surface or an invisibly thin island, we interpolate the label of the surface based on the neighboring irrational surfaces (see part III of Fig. 1).

Finally, we apply the developed technique to an LHD vacuum configuration with the $m / n=1 / 1$ island, where $B_{0}=3 \mathrm{~T}$ and $R_{0}=3.6 \mathrm{~m}$. The Poincare plots of the field lines are shown in Fig. 14, where the vacuum magnetic field is calculated by using the Biot-Savart law [25-27]. Figure 15 shows the result of the labeling of the configuration. In this figure, the green line represents the outside

Table 1 Success rate of the simple method of ordering the Poincaré points. The result is obtained in the $m / n=1 / 1$ island in the test configuration. On the other hand, the developed method always succeeds.

\begin{tabular}{c|c} 
Number of points & Success rate $(\%)$ \\
\hline 50 & 3.0 \\
100 & 46.0 \\
200 & 78.0 \\
500 & 93.0
\end{tabular}

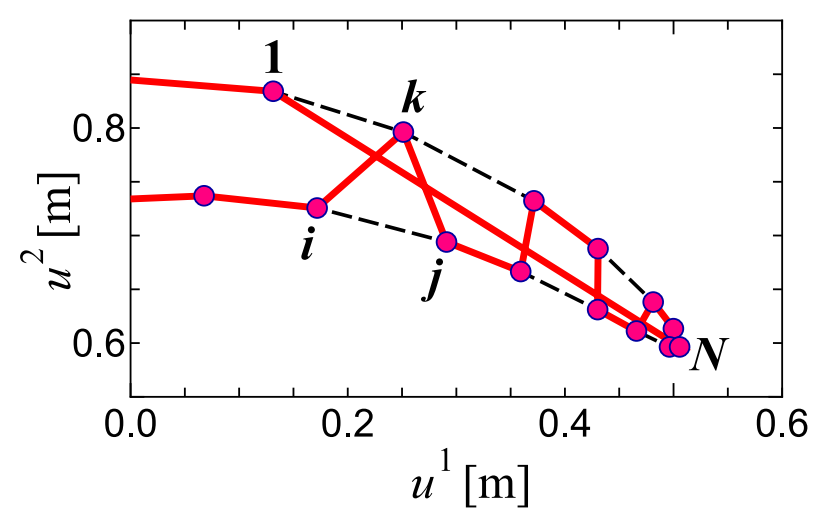

Fig. 13 A result of the simple method of ordering the Poincaré points in the test configuration shown in Fig. 12. The Red line represents the failure to connect each point and the dashed line represents the true solution. Because $L(i, j)>$ $L(i, k)$, the ordering fails. Note that point $N$ has to be connected to point 1 in order to close the curve. 


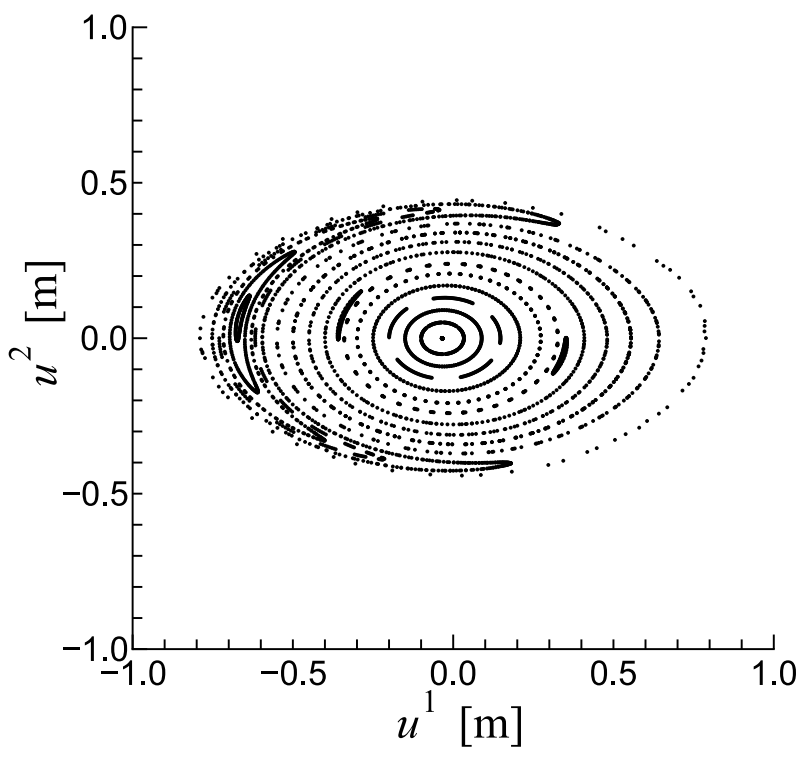

Fig. 14 Poincaré plots of field lines in the LHD vacuum configuration with the $m / n=1 / 1$ island.

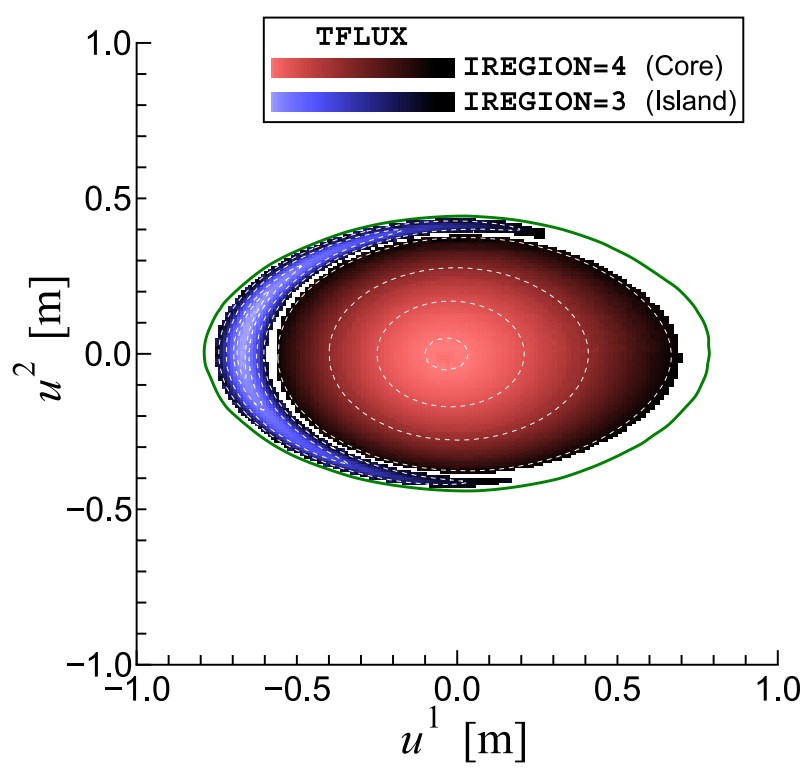

Fig. 15 Label of the magnetic flux-surfaces in the LHD configuration with the $m / n=1 / 1$ island on a poloidal cross section $\left(u^{3}=\pi / 10\right)$. The labels, IREGION and TFLUX, are indicated by colors and their hues, respectively. The green line represents the outside region which is very narrow, and the flux-surfaces in the exterior of the line are not visible under the current level of calculation accuracy.

region which is very narrow, thus magnetic flux-surfaces in the exterior of the line are not visible under this calculation accuracy. We have succeeded in labeling the magnetic flux-surfaces in a configuration containing the island. A radial profile of the labeling is shown in Fig. 16. The profile represents the value of TFLUX in the core and island regions at $u^{2}=Z=0$ and $u^{3}=\pi / 10$, respectively. The

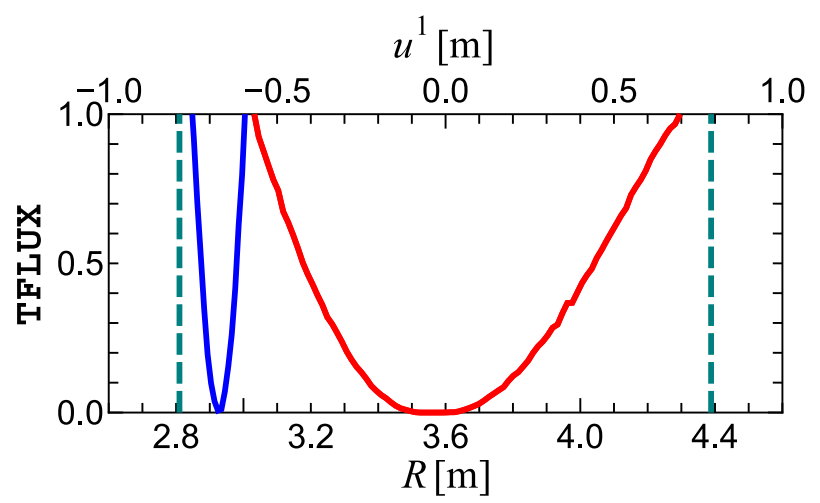

Fig. 16 Radial profile of the labeling in the LHD configuration containing the $m / n=1 / 1$ island as shown in Fig. 15 . The figure indicates the profile of the label TFLUX at $u^{2}=Z=0$ and $u^{3}=\pi / 10$. The red line represents the profile in the core region (IREGION $=4$ ) and the blue line the island region (IREGION $=3$ ). Note that in this figure the minimum of TFLUX in the island region differs slightly from zero. The green dashed line indicates the position of the outside region (IREGION $=1$ ). The ergodic region (IREGION $=2$ ) exists in the gaps of the profile. At $R \simeq 3.25$ and $3.95 \mathrm{~m}(Z=0)$, the profile crosses an invisibly small island with $m / n=2 / 1$; see also Fig. 14.

outside region is indicated by the green dashed line, and the ergodic region exits on the gap between the other three regions.

\section{Summary}

The labeling of magnetic flux-surfaces is needed for transport analysis in non-axisymmetric magnetic field configurations containing magnetic islands. Frequently, labeling the flux-surfaces is difficult because of their complexity. We have developed a computational technique for labeling the magnetic flux-surfaces by applying a simulated annealing method. This algorithm finds the global extremum. The primary challenge is the ordering of the Poincaré points of field lines on a poloidal cross section in order to identify the closed magnetic flux-surfaces. In the developed technique, we label the flux-surfaces in terms of two labels describing the regions of the magnetic field structure (IREGION), and the values of the toroidal magnetic fluxes (TFLUX). Of course, a magnetic coordinate system can be locally constructed on the island region by using the techniques of the present paper and Refs. [15-18].

Using the technique developed here, the flux-surface average can be obtained wherever closed flux-surfaces exist; for example, the flux-surface average of a function $\Phi(\boldsymbol{x})$ is given as

$$
\langle\Phi(\boldsymbol{x})\rangle \simeq \frac{1}{\Delta V} \iiint_{\Delta V} \Phi(\boldsymbol{x}) \mathrm{d}^{3} x,
$$

where $\Delta V$ is the volume of a small shell which lies between two neighboring flux-surfaces determined by the devel- 
oped technique. Note that in an ergodic region (IREGION $=2$ ), the average is given as

$$
\langle\Phi(\boldsymbol{x})\rangle \simeq \frac{1}{V_{2}} \iiint_{V_{2}} \Phi(\boldsymbol{x}) \mathrm{d}^{3} x,
$$

where $V_{2}$ is the total volume of an ergodic region which is assumed to be narrow. This average may be useful in analyzing the transport phenomena in terms of the neoclassical transport theory. Neoclassical transport analysis is frequently carried out on magnetic coordinates [28-31]. However, in an LHD equilibrium having the $m / n=1 / 1$ island, there does not exist a magnetic coordinate system along the flux-surfaces in both the core and island regions. Therefore, we are currently developing a transport simulation code based on techniques without magnetic coordinates [32-34]. The results of transport analysis in/around the island region will be reported in the near future.

\section{Acknowledgments}

We are grateful to Professor H. Sugama for his useful suggestions. This work was performed with the support and under the auspices of the NIFS Collaborative Research Program NIFS06KNXN065, NIFS06KTAT028, NIFS05 KNXN039, NIFS00KTAT002, and NIFS04KLDD001, and was also supported by MEXT. HAITEKU(2004).

[1] S. Hamada, Nucl. Fusion 2, 23 (1962).

[2] A.H. Boozer, Phys. Fluids 23, 904 (1980).

[3] N. Nakajima, J. Todoroki and M. Okamoto, Kakuyugo Kenkyu 68, 395 (1992).

[4] H. Grad, Phys. Fluids 10, 137 (1967).

[5] T. Hayashi, Theory of Fusion Plasmas (Società Italiana di Fisica, Bologna, 1989) p.11.

[6] K. Harafuji, T. Hayashi and T. Sato, J. Comput. Phys. 81, 169 (1989)

[7] R. Kanno, T. Hayashi and M. Okamoto, Nucl. Fusion 45, 588 (2005).

[8] S.R. Hudson and R.L. Dewar, Phys. Lett. A 226, 85 (1997).

[9] R.L. Dewar, S.R. Hudson and P.F. Price, Phys. Lett. A 194, 49 (1998).

[10] J. Todoroki, J. Phys. Soc. Jpn. 58, 3979 (1989).

[11] J. Todoroki, J. Phys. Soc. Jpn. 63, 2168 (1994).

[12] M. Kurata and J. Todoroki, J. Plasma Fusion Res. SERIES 1, 491 (1998).
[13] K. Ida, N. Ohyabu, T. Morisaki et al., Phys. Rev. Lett. 88, 015002 (2002).

[14] K. Ida, S. Inagaki, N. Tamura et al., Nucl. Fusion 44, 290 (2004).

[15] G. Kuo-Petravic, A.H. Boozer, J.A. Rome and R.H. Fowler, J. Comput. Phys. 51, 261 (1983).

[16] J.A. Rome, J. Comput. Phys. 82, 348 (1989).

[17] P.H. Fowler, R.N. Morris, J.A. Rome and K. Hanatani, Nucl. Fusion 30, 997 (1990).

[18] H. Kikuchi, H. Ueno, M. Aizawa et al., Proceedings of the First International Toki Conference on Plasma Physics and Controlled Nuclear Fusion (National Institute for Fusion Science, Toki, 1990) p.183.

[19] S. Kirkpatrick, C.D. Gelatt and M.P. Vecchi, Science 220, 671 (1983).

[20] V. Černý, J. Optim. Theory Appl. 45, 41 (1985).

[21] E.L. Lawler, J.K. Lenstra, A.H.G. Rinnooy Kan and D.B. Shmoys (Ed), The Traveling Salesman Problem: A Guided Tour of Combinatorial Optimization (John Wiley \& Sons Ltd, Eastbourne, 1985).

[22] C.K. Koelbel, D.B. Loveman, R.S. Schreiber, G.L. Steele Jr. and M.E. Zosel, The High Performance Fortran Handbook (MIT Press, Cambridge Massachusetts, 1993).

[23] W.H. Press, B.P. Flannery, S.A. Teukolsky and W.T. Vetterling, Numerical Recipes in C (Cambridge University Press, New York, 1986).

[24] N. Metropolis, A. Rosenbluth, M. Rosenbluth, A. Teller and E. Teller, J. Chem. Phys. 21, 1087 (1953).

[25] J. Todoroki, Kakuyugo Kenkyu 57, 318 (1987) [in Japanese].

[26] J. Todoroki, Kakuyugo Kenkyu 63, 271 (1990) [in Japanese].

[27] J. Todoroki, Jpn. J. Appl. Phys. 43, 1209 (2004).

[28] W.X. Wang, N. Nakajima, M. Okamoto and S. Murakami, Plasma Phys. Control. Fusion 41, 1091 (1999).

[29] M. Okamoto, N. Nakajima, S. Satake and W.X. Wang, J. Plasma Fusion Res. 78, 1344 (2002).

[30] S. Satake, M. Okamoto, N. Nakajima, H. Sugama and M. Yokoyama, Plasma Fusion Res. 1, 002 (2006).

[31] P. Helander and D.J. Sigmar, Collisional Transport in Magnetized Plasmas (Cambridge University Press, Cambridge, 2002).

[32] R. Kanno, S. Jimbo, H. Takamaru and M. Okamoto, J. Plasma Fusion Res. SERIES 6, 527 (2004).

[33] S. Jimbo, R. Kanno, H. Takamaru and M. Okamoto, J. Plasma Fusion Res. 80, 649 (2004).

[34] S. Jimbo, R. Kanno, H. Takamaru, S. Satake and M. Okamoto, Nucl. Fusion 45, 1534 (2005). 